\title{
Diagnostic Value of Bone Marrow Cell Morphology in Visceral Leishmaniasis-Associated Hemophagocytic Syndrome of Two Cases
}

SHULAN SHI ( $\square$ shishulan_job@163.com )

kunming children's hospital

HENG ZHAO

Institute od Medical Biology Chinese Academy of Medical Sciences

MINGBIAO MA

The Kunming Children's Hospital

XIAOJUAN LI

The Kunming Children's Hospital

JIXU

The Kunming Children's Hospital

BENGJIANG ZHOU

Kunming Medical University HaiYuan College

HONGCHAO JIANG

The Kunming Children's Hospital

\section{Case Report}

Keywords: bone marrow cell morphology, visceral leishmaniasis, hemophagocytic syndrome, infant

Posted Date: October 12th, 2021

DOl: https://doi.org/10.21203/rs.3.rs-889416/v1

License: (9) (i) This work is licensed under a Creative Commons Attribution 4.0 International License. Read Full License 


\section{Abstract}

Background: Visceral leishmaniasis related-hemophagocytic lymphohistiocytosis (VL-HLH) is a hemophagocytic syndrome caused by Leishmania infection. VL-HLH is rare, especially in nonendemic areas where the disease is severe, and mortality rates are high. The key to diagnosing VL-HLH is to find the pathogen; therefore, the Leishmania must be accurately identified for timely clinical treatment.

\section{Case presentation}

We retrospectively analyzed the clinical data, laboratory examination results and bone marrow cell morphology of two children with VL-HLH diagnosed via bone marrow cell morphology between July 2017 and January 2021 at Kunming Children's Hospital of Yunnan, China.Two cases suspected of having malignant tumors at other hospitals and who had undergone ineffective long-term treatment were transferred to Kunming Children's Hospital. They had repeated fevers, pancytopenia, hepatosplenomegaly, hypertriglyceridemia, and hypofibrinogenemia over a long period and met the HLH2004 standard. Their HLH genetic test results were negative, and primary HLH was excluded. Both children underwent chemotherapy as per the HLH-2004 chemotherapy regimen , but it was ineffective and accompanied by serious infections. We found Leishmania amastigotes in their bone marrow via morphological examination of their bone marrow cells, which showed hemophagocytic cells; thus, the children were diagnosed with VL-HLH. After being transferred to a specialty hospital for treatment, the condition was well-controlled.

Conclusion: Morphological examination of the bone marrow cells played an important role in diagnosing VL-HLH. When clinically diagnosing secondary HLH, VL-HLH should be considered in addition to common pathogens, especially in patients for whom HLH-2004 chemotherapy regimens are ineffective. For infants and young children, bone marrow cytology examinations should be performed several times and as early as possible to find the pathogens to reduce potential misdiagnoses.

\section{Background}

Hemophagocytic syndrome (HPS), also known as hemophagocytic lymphohistiocytosis (HLH), is divided into primary and secondary forms. The secondary etiology is complex and can be caused by infection, malignant tumors, and autoimmune diseases.Visceral leishmaniasis related-hemophagocytic lymphohistiocytosis (VL-HLH) is very rare in childhood, especially in nonendemic areas. The disease is severe with high mortality rates of up to $100 \%$ without early diagnosis and treatment [1]. Therefore, the leishmaniasis-associated pathogen must be rapidly and accurately identified for clinical and timely treatment. Here, we report two young patients with VL-HPS diagnosed via bone marrow cell morphology at the Children's Hospital of Kunming, Yunnan, China in the past 5 years.

\section{Case Presentation}

\section{Case 1}


A 2-year-old girl from Weining, Guizhou, China, presented in July 2017 with a repeated irregular fever lasting 3 months and reaching $39^{\circ} \mathrm{C}-40^{\circ} \mathrm{C}$. After the illness, she was hospitalized at a local hospital for 2 months. Her whole blood cells decreased progressively, and she underwent symptomatic and supportive treatment, including meropenem, vancomycin, piperacillin, tazobactam, gamma globulin, methylprednisolone, cefoperazone, sulbactam, Taineng, and a blood transfusion. She continued to have repeated fevers, coughing with sputum, abdominal distension, anorexia, and fatigue. She had a history of mosquito bites and contact with a domestic dog 1 month before onset as well as a history of Epstein-Barr virus-related hemophagocytic syndrome.

\section{Case 2}

A 9-month-old girl from Zhaotong City, Yunnan Province, China, who had been living in Zhouqu County, Gansu Province, from July to September 2020 was admitted to our hospital. During this period, the infant had a history of mosquito bites that resolved after 3 months. She had diarrhea for half of December 2020 , with a subsequent irregular fever lasting 1 month and reaching $39^{\circ} \mathrm{C}-40^{\circ} \mathrm{C}$ and decreased blood cells in the peripheral blood. She did not recover at her local hospital and was thus transferred to Kunming Children's Hospital.

Both patients had hepatosplenomegaly, hypertriglyceridemia, and hypofibrinogenemia. Tables 1-5 show the relevant results. The results of the hemophagocytic cells in both infants' bone marrow while at the other hospital met the HLH-2004 standard [2]. The results of the primary HPS-related genetic test were negative and ruled out congenital HPS. All results were in accordance with the HPS-2004 chemotherapy regimen [2].

According to HLH-2004 chemotherapy regimen : $₫$ Dexamethasone: from Day 2 after admission, 10 $\mathrm{mg} /\left(\mathrm{m}^{2} . \mathrm{d}\right)$ for 2 weeks, $5 \mathrm{mg} /\left(\mathrm{m}^{2} . \mathrm{d}\right)$ for 4 days; $\otimes$ Etoposide: from Day 6 after admission, twice a week, 5 $\mathrm{mg} / \mathrm{kg}$ each time, a total of 4 times; $\otimes$ Other support treatment: blood and immunoglobulin transfusion,recombinant human granulocyte stimulating factor. However, after standardized HLHassociated chemotherapy, the symptoms did not significantly disappear, and the patients still had fevers and severe infection. Both patients' bone marrow cell morphology was re-examined at Kunming Children's Hospital. The pathogens of Kala-azar and Leishmania amastigotes were found, along with hemophagocytic cells; thus, VL-HPS was diagnosed. However, because our hospital lacks the drugs to treat this disease, the patients were transferred to a specialty hospital. Telephone interviews confirmed that after receiving symptomatic medication at the specialty hospital, the patients' conditions quickly improved. Both patients recovered and were discharged.

\section{Laboratory inspection}


Table 1

Basic conditions of two patients with Visceral leishmaniasis

\begin{tabular}{|lll|}
\hline General conditions & Case 1 & Case 2 \\
\hline Age & 2 years & 9 months \\
\hline Sex & female & female \\
\hline Onset month & July & November \\
\hline History of mosquito bites & Yes & Yes \\
\hline Fever & $39^{\circ} \mathrm{C}-40^{\circ} \mathrm{C}$ & $39.5^{\circ} \mathrm{C}-40^{\circ} \mathrm{C}$ \\
\hline Cough & Yes & No \\
\hline Reaction & Poor & Poor \\
\hline Course of illness & 3 months & 2 months \\
\hline History of epidemiology & Yes & Yes \\
\hline
\end{tabular}

\section{Pathogenic examination}

The results of the examination for related pathogens revealed the kala-azar pathogen/Leishmania amastigotes in the bone marrow of both patients (Figs. 1 and 2).Leishmania amastigotes can be seen inside and outside of phagocytes, Its shape is round and oval, with a diameter of about 2 to 5 um, The cytoplasm is light blue, with a large round nucleus inside, and the nucleus is purplish red.Beside the nucleus, a small, rod-shaped, and darkly colored moving matrix can be seen.The morphological characteristics were consistent with those of Leishmania amastigotes. Hemophagocytic cells were easily seen in the bone marrow of both patients (Figs. 3 and 4.2). One child was infected with the Epstein-Barr virus but tested negative for other pathogens (Table 2). 
Table 2

Pathogen detection in two patients with Visceral leishmaniasis

\begin{tabular}{|lll|}
\hline Etiological examination & Case 1 & Case 2 \\
\hline Epstein-Barr virus & $2.82 \mathrm{E}^{\wedge} 03$ & negative \\
\hline Cytomegalovirus & negative & negative \\
\hline Rubella virus & negative & negative \\
\hline Influenza virus & negative & negative \\
\hline Respiratory syncytial virus & negative & negative \\
\hline Adenovirus & negative & negative \\
\hline Legionella pneumophila & negative & negative \\
\hline Mycoplasma pneumoniae, Chlamydia & negative & negative \\
\hline Q fever, rickettsia & negative & negative \\
\hline Blood culture & negative & negative \\
\hline Bone marrow cytology & & \\
\hline Leishmania amastigotes & positive & positive \\
\hline Hemophagocytic cells & easily seen & easily seen \\
\hline Hemophagocytic-related genes & negative & negative \\
\hline Acute and chronic leukemia immunophenotyping & negative & negative \\
\hline
\end{tabular}

Table 3. Blood routine indexes, infection indexes and biochemical results for both patients 


\begin{tabular}{|c|c|c|c|c|c|}
\hline \multirow{2}{*}{$\begin{array}{l}\text { Detection index } \\
\text { Characteristics }\end{array}$} & \multirow[t]{2}{*}{$\begin{array}{l}\text { Normal } \\
\text { range }\end{array}$} & \multicolumn{2}{|c|}{ On admission } & \multicolumn{2}{|c|}{$\begin{array}{l}\text { After treatment for } 14 \\
\text { days }\end{array}$} \\
\hline & & Case 1 & Case 2 & Case 1 & Case 2 \\
\hline White blood cell count, $\times 109 / \mathrm{L}$ & $4.0-10.0$ & 2.0 & 4.5 & 3.4 & 3.9 \\
\hline Red blood cell count, $\times 10^{12} / \mathrm{L}$ ) & $4.0-5.5$ & 2.0 & 3.8 & 3.3 & 3.3 \\
\hline Hemoglobin, g/L & $97-141$ & 69.0 & 94.0 & 91.0 & 97.0 \\
\hline Platelet count, $\times 109 / \mathrm{L}$ & $100-300$ & 35.0 & 44.0 & 66.0 & 28.0 \\
\hline $\begin{array}{l}\text { High-sensitivitC-reactive } \\
\text { protein(mg/L) }\end{array}$ & $0.5-10.0$ & 151.8 & 198.3 & 85.3 & 16.3 \\
\hline Procalcitonin $(\mathrm{ng} / \mathrm{ml})$ & $0-0.25$ & 5.4 & 2.3 & 3.5 & 1.8 \\
\hline Ferritin $(\mu \mathrm{g} / \mathrm{L})$ & $7.0-142.0$ & 40000.0 & 69445.0 & 1886.0 & 2000.0 \\
\hline Fibrinogen $(\mathrm{g} / \mathrm{L})$ & $2.0-4.0$ & 1.2 & 0.9 & 1.1 & 1.4 \\
\hline Alanine aminotransferase (U/L) & $0-40.0$ & 75.0 & 178.0 & 41.0 & 149.0 \\
\hline Total protein (g/L) & $55.0-76.0$ & 62.2 & 55.7 & 62.2 & 65.1 \\
\hline Albumin $(\mathrm{g} / \mathrm{L})$ & $39.0-54.0$ & 24.7 & 29.2 & 24.7 & 30.9 \\
\hline Globulin (g/L) & $12.0-34.0$ & 37.5 & 36.5 & 37.5 & 34.2 \\
\hline Lactate dehydrogenase (U/L) & $109.0-245.0$ & 2700.0 & 2648.0 & 256.0 & 1803.8 \\
\hline Triglycerides (mmol/L) & $1.70-2.30$ & 4.2 & 4.5 & 2.0 & 2.5 \\
\hline
\end{tabular}

Routine blood test, infection index and biochemical examination resultsdecreased significantly but remained higher than the normal reference range. Alanine aminotransferase, lactate dehydrogenase and a-hydroxybutyric acid were increased, and albumin was decreased in both children (Table 3).Both children had reductions in whole blood cells or in two lines before treatment. The infection indicators (i.e., highsensitivity C-reactive protein, procalcitonin, and ferritin) were significantly increased, and fibrinogen was significantly reduced. After 14 days of treatment per the HLH-2004 chemotherapy regimen, their routine blood indexes changed little, and their infection indexes

\section{Discussion And Conclusions}

HPS is a life-threatening disease caused by excessive inflammation and multiple organ dysfunction, resulting in uncontrollable lymphocyte and macrophage activation and proliferation [3]. HLH is divided into primary and secondary forms. Infection is the most common cause of secondary HLH[4]. VL-HLH is very rare in childhood and has a high mortality rate if not diagnosed and treated early[1].

Kala-azar is caused by Leishmania, and sandflies are the main transmission vector. The infectious agents of this disease are mainly the patients and dogs. The disease is transmitted between humans and 
animals directly or between humans and animals via blood sucking by sandflies [5-7]. The disease has obvious regional characteristics. VL is scattered throughout six western provinces in China and Xinjiang, Gansu, Sichuan, and Shaanxi [8]. The two cases reported herein were from Weining County, Guizhou, and Yunnan after moving from Zhouqu, Gansu. Both children had lived in epidemic areas and had histories of sandfly bites from June to September (the sandfly breeding season) before disease onset. Weining County in Guizhou and Zhouqu County in Gansu Province are both areas where kala-azar is spreading [8]. The epidemiological histories of both children were clear.

Both children had long-term irregular fevers, with the highest body temperature exceeding $40^{\circ} \mathrm{C}$, pancytopenia, and hepatosplenomegaly. Because of these clinical manifestations, the diseases were initially misdiagnosed as malignant hematological diseases. Neither child recovered after long-term treatment with drugs at other hospitals, and both had severe infections. VL-HLH is easily misdiagnosed in nonendemic areas because VL manifestations are very similar to those of hematological malignancies. VL symptoms also include a long-term irregular fever, hepatosplenomegaly, and pancytopenia. Additionally, VL has a rapid onset and progression. Early symptoms are atypical with many complications; thus, it is easily misdiagnosed [9], especially when combined with Epstein-Barr viral infections, leading clinicians to think that it is Epstein-Barr virus-associated HLH. Many clinicians have insufficient knowledge and no clinical experience with VL, especially in nonendemic areas. Furthermore, laboratory physicians often lack knowledge of the Leishmania amastigotes as VL pathogens because amastigotes in the bone marrow of patients with VL mainly grow and multiply in macrophages and can be seen in the bone marrow when many phagocytic cells contain Leishmania amastigotes. At same time these phagocytes may also contain platelets, red blood cells, and white blood cells, and if the laboratory technicians are unfamiliar with Leishmania amastigotes or do not read the results carefully, they may mistake them for platelets. Many reports have found that kala-azar is often misdiagnosed owing to clinicians' and technicians' lack of knowledge of Leishmania amastigotes [10]. The morphologies of Leishmania, Penicillium marneffei, and histoplasma have many similarities and are easily confused. Clinicians and technicians must be familiar with the morphological characteristics of various pathogens and the differences between them. No Leishmania amastigotes were found in either child via bone marrow cell morphology at the previous hospital; thus, the children were misdiagnosed with hematological malignancies. The key to diagnosing these children is to detect the Leishmania amastigotes via bone marrow cell morphology combined with their epidemiological histories. To diagnose $\mathrm{HLH}$ secondary to kala-azar, finding the pathogen in the bone marrow is the most reliable diagnostic criterion.

VL-HLH-associated mortality is relatively high. If HLH treatment is ineffective, clinicians should consider whether the HLH is secondary to VL. Both patients in this study underwent 14 days of chemotherapy according to the HLH-2004 chemotherapy regimen, but the chemotherapeutic effect was unsatisfactory, their clinical symptoms did not significantly improve, and their liver and kidney functions of did not recover as per the related infection indicators. After the disease was clearly diagnosed, they received symptomatic treatment, and the disease was quickly controlled. Both patients recovered and were discharged from the hospital. When clinically diagnosing $\mathrm{HLH}$, clinicians should actively search for the 
cause. If standard treatment for HLH is ineffective, detailed epidemiological histories should be taken, bone marrow cytology examinations should be performed quickly, and HLH secondary to VL should be ruled out.

In summary, bone marrow cell morphological examinations play a vital role in diagnosing VL-HLH. When secondary HLH is diagnosed clinically, common pathogens and VL-HLH should both be considered, especially in infants and young children who could not be treated satisfactorily as per the HLH-2004 regimen. Detailed epidemiological histories should be taken, and their bone marrow cytology should be examined multiple times as soon as possible to find the pathogen and reduce misdiagnoses. Clinicians and technicians should be familiar with the morphological characteristics of Leishmania to provide timely and accurate diagnoses.

\section{Abbreviations}

HPS, hemophagocytic syndrome; VL, visceral leishmaniasis; HLH, hemophagocytic lymphohistiocytosis; VL-HLH, visceral leishmaniasis related-hemophagocytic lymphohistiocytosis

\section{Declarations}

Authors' contributions

S-LS and HZ wrote the manuscript and carried out the analysis.M-BM,X-JL and JX investigated these cases.B-JZ and H-CJ designed and supervised this study. All authors read and approved the final manuscript.

Authors' information

Not applicable.

Funding

the Association Foundation Program of Yunnan Science and Technology Department and Kunming Medical University (grant no. 2019FE001-103 ), Yunnan Health Training Project of High Level Talents (grant no. D-2017053 ), Top Young Experts Training Project for the Academy and Technology in Kunming and Yunnan Province (grant no. 202005AC160066), Postdoctoral Training Program of Yunnan Province (grant no. Ynbh19035) and Natural Science Foundation of Yunnan Province (grant no. 2019-1-C25318000002240 ).

Availability of data and materials

All original data and materials are available from the corresponding author upon request

Ethics approval and consent to participate 
The present study was approved by the Ethics Committee of the Affiliated Children's Hospital of Kunming Medical University (Kunming, China), and written informed consent was obtained from each donor's guardian.

Consent for publication

The case described here provided written consent for the publication of this case report.

Competing interests

The authors declare no competing interests.

Author details

${ }^{1}$ Institute of Pediatrics, The Kunming Children's Hospital, Kunming, Yunnan $650228{ }^{2}$ Institute of Medical Biology Chinese Academy of Medical Sciences, Kunming, ${ }^{3}$ Yunnan 650031,Kunming Mdeical Univesity HaiYuan College

${ }^{4}$ Yunnan Key Laboratory of Children's Major Disease Research, Kunming

\section{References}

1. Sundar S, Singh OP, Molecular Diagnosis of Visceral Leishmaniasis Mol Diagn Ther.2018, 22(4): 443-57. https:// doi.org/10.1007/s40291-018-0343-y

2. Henter Jl, Horne A, Aricó M, et al. HLH-2004: diagnostic and therapeutic guidelines for hemophagocytic lymphohistiocytosis. Pediatr Blood Cancer 2007;48(2):124-31.https:// doi.org/10.1002/pbc.21039.

3. Janka GE. Familial and acquired hemophagocytic lymphohistiocytosis. Annu Rev Med 2012;63:233-46.https:// doi.org/10.1146/annurev-med-041610-134208.

4. Janka G, Imashuku S, Elinder G, et al. Infection- and malignancy-associated hemophagocytic syndromes. Secondary hemophagocytic lymphohistiocytosis. Hematol Oncol Clin North Am 1998;12:435-44. https:// doi.org/ 10.1016/s0889-8588(05)70521-9.

5. Wang JY, Gao CH, Yang YT, et al. An outbreak of the desert sub-type of zoonotic visceral leishmaniasis in Jiashi, Xinjiang Uygur Autonomous Region, People's Republic of China. Parasitol Int 2010,59(3):331-37. https:// doi.org/ 10.1146/annurev-med-041610-134208.

6. Lu HG, Zhong L, Guan LR, et al. Separation of Chinese Leishmania isolates into five genotypes by kinetoplast and chromosomal DNA heterogeneity. Am J Trop Med Hyg 1994;50(6):763-70. https:// doi.org/10.4269/ajtmh.1994.50.763.

7. Wang JY, Ha Y, Gao CH, et al. The prevalence of canine Leishmania infantum infection in western China detected by PCR and serological tests. Parasit Vectors 2011;9(4):69-77. https:// doi.org/ 10.1186/1756-3305-4-69. 
8. Zheng C, Wang L, Li Y, et al. Visceral leishmaniasis in northwest China from 2004 to 2018: a spatiotemporal analysis. Infect Dis Poverty 2020;9(1):165. https://doi.org/10.1186/s40249-02000782-4.

9. Zhao S, Li Z, Zhou S, et al. Epidemiological feature of visceral leishmaniasis in China, 2004-2012. Iran J Public Health 2015;44(1):51-9.

10. Schwing A, Pomares $C$, Majoor A, et al. Leishmania infection: misdiagnosis as cancer and tumorpromotingpotential.ActaTrop 2019;9:197.https://doi.org/10.1016/j.actatropica.2018.12.010.

\section{Figures}


\section{Figure 1}

Case 1: Leishmania amastigotes inside(fig.a) and outside (fig.b)of phagocytes in the bone marrow ( Wright-Giemsa,×1000)

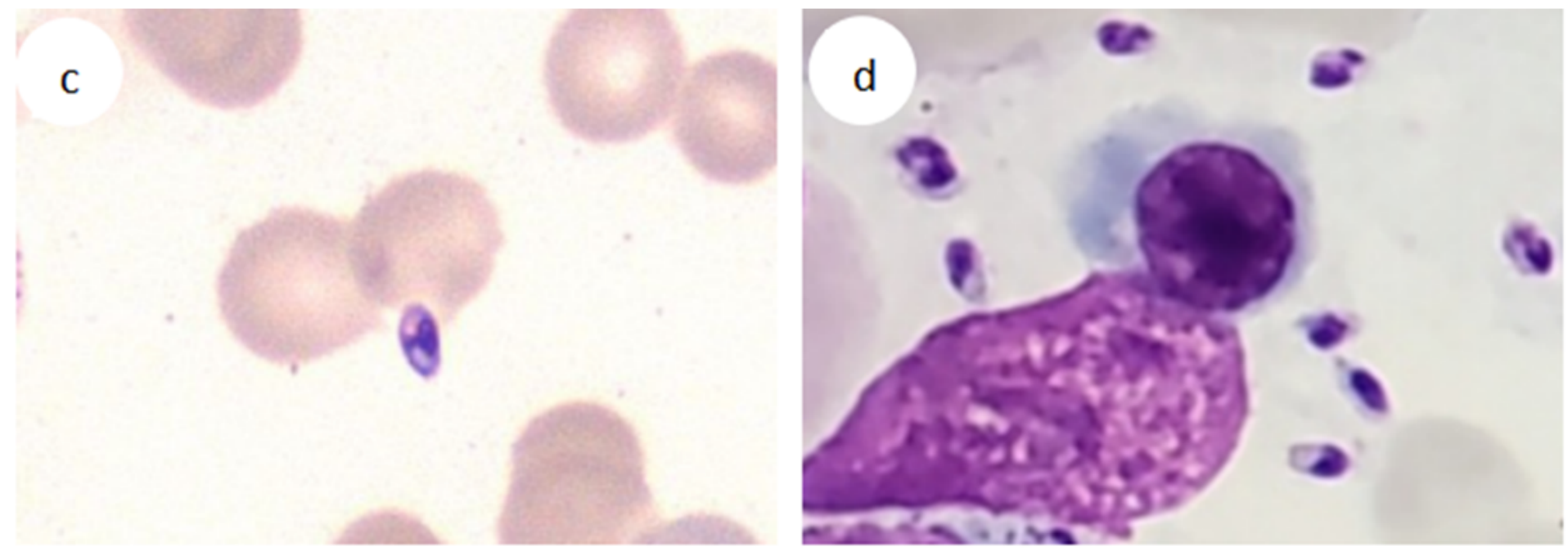

Figure 2 
Leishmania amastigotes scattered (fig.c) and piles (fig.d) in the bone marrow of case 2 (WrightGiemsa,×1000)
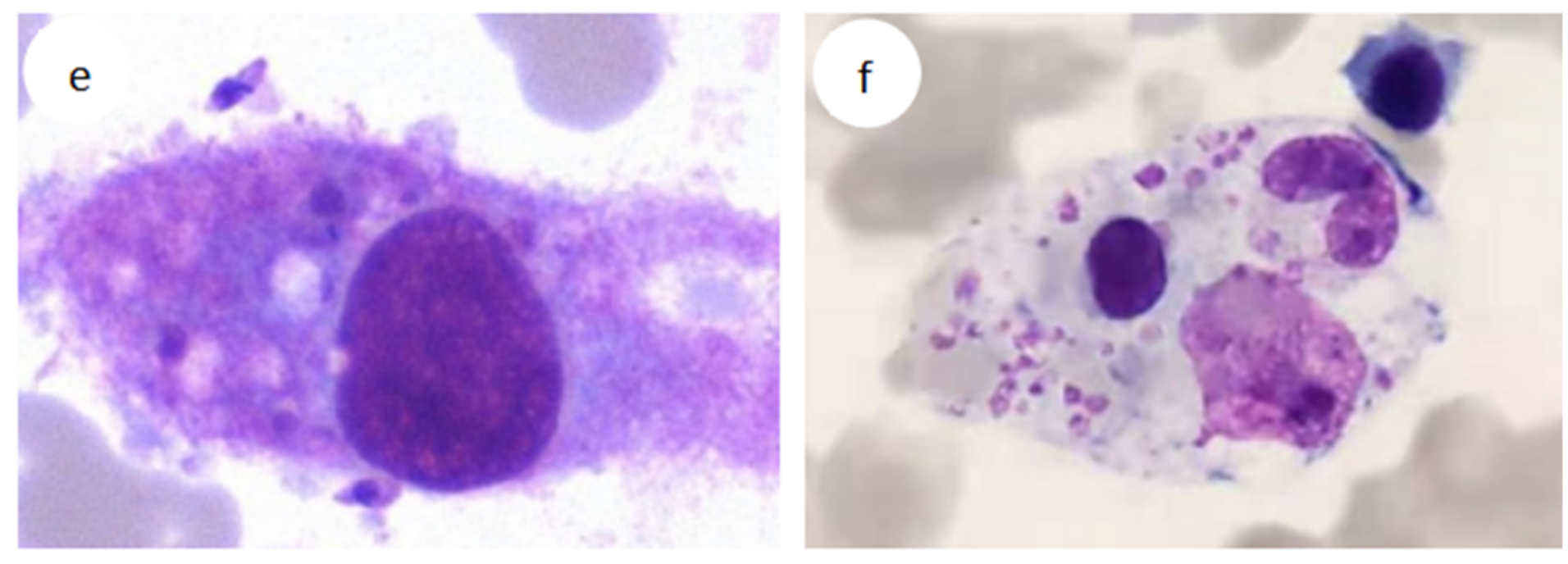

\section{Figure 3}

Hemophagocytic cells in the bone marrow (case 1, fig.e; case 2, fig.f)(Wright-Giemsa, $\times 1000$ ) 